\title{
Laparoscopic Pancreas-Sparing Subtotal Duodenectomy in Gastrointestinal Stromal Tumor of the Duodenum
}

\author{
Sung Hoon Kim, MD , Chang Moo Kang, $\mathrm{MD}^{2,3}$, and Woo Jung Lee, MD,PhD ${ }^{2,3}$ \\ ${ }^{1}$ Department of Surgery, Wonju Christian Hospital, Yonsei University Wonju College of Medicine, Wonju, Korea; \\ ${ }^{2}$ Division of Hepatobiliary and Pancreas, Department of Surgery, Yonsei University College of Medicine, Seoul, Korea; \\ ${ }^{3}$ Pancreaticobiliary Cancer Clinic, Institute of Gastroenterology, Yonsei University Health System, Seoul, Korea
}

\begin{abstract}
Background. The duodenum is a rare origin for gastrointestinal stromal tumors (GISTs). ${ }^{1,2}$ A decision of pancreatoduodenectomy or limited resection is a dilemma for surgeons. Recent reviews have suggested that types of surgery did not influence prognosis and limited resection was indicated for small GIST located some distance away from the ampulla of Vater (AOV). ${ }^{3,4}$ However, a laparoscopic, pancreas-preserving, subtotal duodenectomy was rarely performed. ${ }^{5,6}$

Methods. A 20-year-old female was referred to our institution because of a duodenal submucosal mass. Computer tomography and endoscopy revealed a 3.8- $\mathrm{cm}$-sized mass that was $\sim 2 \mathrm{~cm}$ from AOV. A minimally invasive and function-preserving resection was scheduled.

Results. Meticulous dissection of the duodenum from the pancreatic head was a critical point. Even small breakages of vessels could provoke massive bleeding, possibly resulting in the surgeon's view being obstructed, longer operating times, or a decreased chance of performing a minimally invasive and limited resection. Therefore, an
\end{abstract}

Electronic supplementary material The online version of this article (doi:10.1245/s10434-012-2729-0) contains supplementary material, which is available to authorized users.

(C) Society of Surgical Oncology 2012

First Received: 29 August 2011;

Published Online: 3 November 2012

C. M. Kang

e-mail: cmkang@yuhs.ac especially meticulous and careful dissection was performed. An upper gastrointestinal series revealed no leakage, and the patient received a soft diet on postoperative day 3 . The patient was discharged on postoperative day 8. Pathologic examination reported a low-risk GIST group.

Conclusions. Although clearly malignant tumors are not suitable for this approach due to poor oncologic outcomes, laparoscopic pancreas-preserving subtotal duodenectomy is a feasible and effective strategy to treat benign or borderline tumors. This approach will offer successful oncologic results and laparoscopic merits. We feel that this demonstration would advocate clinical feasibility of minimally invasive and function-preserving resections in well-selected duodenal GISTs.

DISCLOSURE Sung Hoon Kim, Chang Moo Kang, and Woo Jung Lee have no conflict of interest or financial ties to disclose.

\section{REFERENCES}

1. Goh BKP, Chow PKH, Kesavan S, Yap WM, Wong WK. Outcome after surgical treatment of suspected gastrointestinal stromal tumors involving the duodenum: is limited resection appropriate? J Surg Oncol. 2008;97:388-91.

2. Winfield RD, Hochwald SN, Vogel SB, Hemming AW, Liu C, Cance WG, Grobmyer SR. Presentation and management of gastrointestinal stromal tumors of the duodenum. Am Surg. 2006; 72:719-22.

3. Cassier PA, Blay JY. Gastrointestinal stromal tumors of the stomach and duodenum. Curr Opin Gastroenterol. 2011;27:571-5.

4. Gervaz P, Huber O, Morel P. Surgical management of gastrointestinal stromal tumours. Br J Surg. 2009;96:567-78.

5. Poves I, Burdio F, Alonso S, Seoane A, Grande L. Laparoscopic pancreas-sparing subtotal duodenectomy. J Pancreas. 2011;12: 62-5.

6. Dholakia C, Gould J. Minimally invasive resection of gastrointestinal stromal tumors. Surg Clin North Am. 2008;88:1009-18. 